\title{
Florística y fisonomía de un matorral xerófilo espinoso intervenido en Punta de Piedras, Municipio Miranda, Estado Zulia, Venezuela
}

\author{
Antonio Vera $^{1 *}$, Maritza Martínez ${ }^{2}$, Yin Ayala ${ }^{1,3}$, Sadieth Montes ${ }^{1,3} \&$ Anderson González ${ }^{1,3}$ \\ 1. Laboratorio de Ecología, Centro de Investigaciones Biológicas, Facultad de Humanidades y Educación, Universidad \\ del Zulia, Apartado 526, Maracaibo 4001-A, Estado Zulia, Venezuela; ajvera68@intercable.net.ve \\ 2. Centro de Investigaciones en Química de los Productos Naturales, Facultad de Humanidades y Educación, Universidad \\ del Zulia, Apartado 526, Maracaibo 4001-A, Estado Zulia, Venezuela \\ 3. Estudiantes de la Maestría en la Enseñanza de la Biología, Facultad de Humanidades y Educación, Universidad del \\ Zulia, Apartado 526, Maracaibo 4001-A, estado Zulia, Venezuela \\ * Autor para la correspondencia
}

Recibido 25-II-2007. Corregido 05-XI-2007. Aceptado 31-VII-2008.

\begin{abstract}
Floristic and physiognomic characteristics of disturbed thorny xerophytic scrubland in Punta de Piedras, Miranda Municipality, Zulia State, Venezuela. We determined the floristic and physiognomic aspects of disturbed thorny xerophytic scrubland in Punta de Piedras, Miranda Municipality, Zulia State, Venezuela. Botanical sampling was done twice monthly (November 2005-June 2006), in both rainy and dry seasons, for a total of 16 samples. The sampling was done in 10 vegetation patches, in an average area of 718,2 $\pm 706,24 \mathrm{~m}^{2}$, and adjacent graminoid floristic components were identified using three $400 \mathrm{~m}^{2}(20 \times 20 \mathrm{~m})$ plots, divided into 4 subplots $\left(20 \times 5 \mathrm{~m}^{2}\right)$ and in these, were delimited 12 subplots $\left(1 \times 1 \mathrm{~m}^{2}\right)$. In addition, human paths were carried out in order to identify other species plant. Thorny xerophytic scrubland showed human disturbances e.g. periodic fires, solid wastes, roads and human settlements and coastal semiarid thorny scrubland characteristics, similar to another Venezuelan localities. Forty-five species, in 22 families were identified, and the Poaceae (6), Mimosaceae (4), Boraginaceae (3), Cactaceae (3), Caesalpinaceae (3), Euphorbiaceae (3) and Fabaceae (3) presented the highest number of species. Human disturbances probably favor the appearance of vegetation patches and secondary grassland formations. In the last, Aristida venesuelae, Aristida pittieri $\mathrm{y}$ Portulaca pilosa showed the highest relative abundances and frequencies values with a summatory of $49,38 \%$ and $46,86 \%$, respectively, while in the patches there were observed two layer: an herbaceous layer (less than 1 $\mathrm{m}$ high) as well as a bush-tree layer (2.63 $\pm 1.37 \mathrm{~m}$ high), comprised mostly of Opuntia wentiana, Piptadenia flava and Jatropha gossypifolia with relative abundances of 32,82, 15,60 and 15,14 \%, respectively. The thorny xerophytic scrubland in Punta de Piedras presents a low number of species; however, the physiognomic characteristics are similar to other undisturbed thorny xerophytic scrublands in Venezuela. Rev. Biol. Trop. 57 (1-2): 271-281. Epub 2009 June 30.
\end{abstract}

Key words: thorny xerophytic scrubland, very dry tropical forest, disturbed ecosystem, floristic inventory, vegetative physiognomy, Venezuela, Zulia.

Las regiones áridas y semiáridas, dentro de las zonas de vida de Venezuela, representan un alto porcentaje del territorio nacional (Castillo et al. 1992), con un total aproximado de 28000 $\mathrm{km}^{2}$ cubiertos por vegetación xerofita espinosa (Ministerio del Ambiente y de los Recursos
Naturales Renovables 1980). Estas regiones comprenden comunidades vegetales tales como matorrales litorales xerófilos en el Cayo Noreste del Refugio de Fauna Silvestre "Cuare", estado Falcón (Huber y Alarcón 1988); montes espinosos tropicales en la Meseta de Mamo, estado 
Vargas y en el cerro Colorado de Cumaná, estado Sucre (Tamayo 1967, Ewel y Madriz 1968, Sarmiento 1976, Castillo et al. 1992, Prieto et al. 2001), cardonales-espinares costaneros en el Parque Nacional Henri Pittier, estados Aragua y Carabobo (Fernández Badillo 1997), bosques xerófilos en la región de Las Peonías, estado Zulia (González 1980) y sabanas xerofíticas en la Goajira venezolana (Zambrano 1994). Algunas de estas formaciones vegetales han sido intervenidas, degradadas, y en muchos casos, han desaparecido bajo la presión urbana, agrícola y ganadera (Ewel y Madriz 1968).

Los bosques secundarios o intervenidos en los trópicos suelen ser definidos como vegetaciones leñosas sucesionales que se desarrollan sobre tierras cuya vegetación original ha sido destruida por actividades humanas (Santana et al. 2002). Entre los factores que afectan la riqueza de especies y la estructura de la vegetación, destacan las perturbaciones antrópicas, las cuales representan el factor de mayor impacto en las comunidades naturales. Una de las consecuencias de las perturbaciones es la división del ambiente natural de bosque en un mosaico de estados sucesionales de la vegetación, lo cual puede determinar la estructura de manchones en las comunidades y la consecuente especialización de las especies a hábitat particulares (López y Ramírez 2004).

Noguera y Ruiz Zapata (2004) informaron para el Cerro La Gruta, estado Aragua, próximo a la población de San Sebastián, una transformación del componente botánico original, correspondiente a un bosque seco, a una vegetación secundaria estructuralmente más simple y pobre florísticamente, representada por un arbustal o matorral. En consecuencia, se ha sugerido la importancia de ampliar el conocimiento de las características morfológicas, ecológicas, fisiológicas y reproductivas de las especies de las comunidades vegetales, como los matorrales xerófilos litorales y los montes espinosos tropicales, a objeto de utilizarlos como fuente potencial de recursos a través de planes de desarrollo sustentable (Castillo et al. 1992).

Por otra parte, en Venezuela, los estudios sobre la caracterización florística y fisonómica de matorrales espinosos intervenidos son escasos.

El objetivo principal de este trabajo es determinar la composición florística y los rasgos fisonómicos de un matorral xerófilo espinoso intervenido en Punta de Piedras, municipio Miranda, estado Zulia, Venezuela.

\section{MATERIALES Y MÉTODOS}

Área de estudio. La investigación se llevó a cabo en una comunidad vegetal ubicada en la zona de vida de Bosque Muy Seco Tropical (Ewel y Madriz 1968) del pueblo de Punta de Piedras a $10^{\circ} 39^{\prime} 52^{\prime \prime} \mathrm{N}$ y 71³1'10" O del municipio Miranda, Costa Oriental del Lago de Maracaibo, estado Zulia, Venezuela (Fig. 1). Dicha comunidad comprende por una parte, un matorral de plantas espinosas en condiciones climáticas semiáridas, distribuidas en manchones,

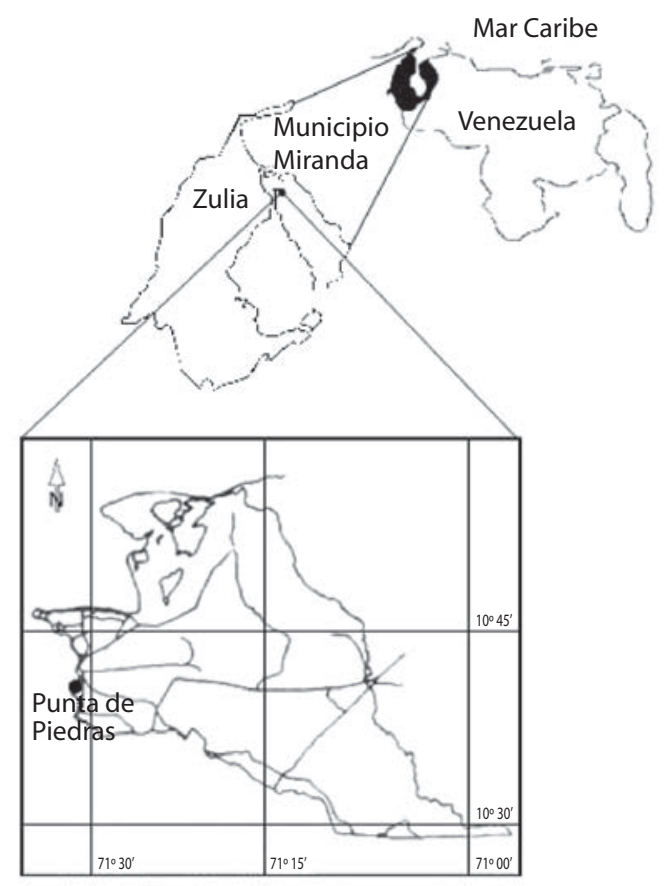

Fig. 1. Localización del área de estudio.

Fig. 1. Localization of study area. 
y por otra, una formación graminiforme; ambos presentan rasgos de vegetación secundaria (no primaria o virgen) producto de la intervención antropogénica en la zona.

Desde el punto de vista climatológico, el área de estudio se caracteriza por presentar dos períodos estacionales bien delimitados: uno seco de diciembre a abril, y otro lluvioso, de mayo a noviembre (Fig. 2). El promedio anual de precipitación varía entre 500 a $1000 \mathrm{~mm}$, lo cual es 2 a 4 veces menor que la evapotranspiración potencial, situación que produce déficit hídrico (Ewel y Madriz 1968).

El área de estudio se localiza próxima a la zona litoral y presenta suelos secos y arcillosoarenosos. De acuerdo a la estación metereológica Hospital de Cabimas, localizada a $30 \mathrm{~km}$ al sur del área de estudio, la temperatura y la precipitación media son de $28.7^{\circ} \mathrm{C}$ y 614.2 $\mathrm{mm}$, respectivamente (Fig. 2).

Muestreo, recolecta e identificación de las especies. Se realizaron dieciséis (16) muestreos quincenales (noviembre 2005 - junio 2006), tanto en el periodo lluvioso como en el seco, para la recolecta de muestras botánicas. Se realizaron muestreos a nivel de 10 manchones, con un área promedio de $718.2 \pm 706.24 \mathrm{~m}^{2}$, en los cuales se encontraba dispuesta la vegetación, para la identificación y conteo de los arbustos y árboles, mientras que para las hierbas de la formación graminiforme, se emplearon tres parcelas de $20 \times 20\left(400 \mathrm{~m}^{2}\right)$. Cada una de éstas se dividió en cuatro subparcelas de 20 x $5\left(100 \mathrm{~m}^{2}\right)$ y a su vez, dentro de ellas, se delimitaron subparcelas de $1 \times 1\left(1 \mathrm{~m}^{2}\right)$. En

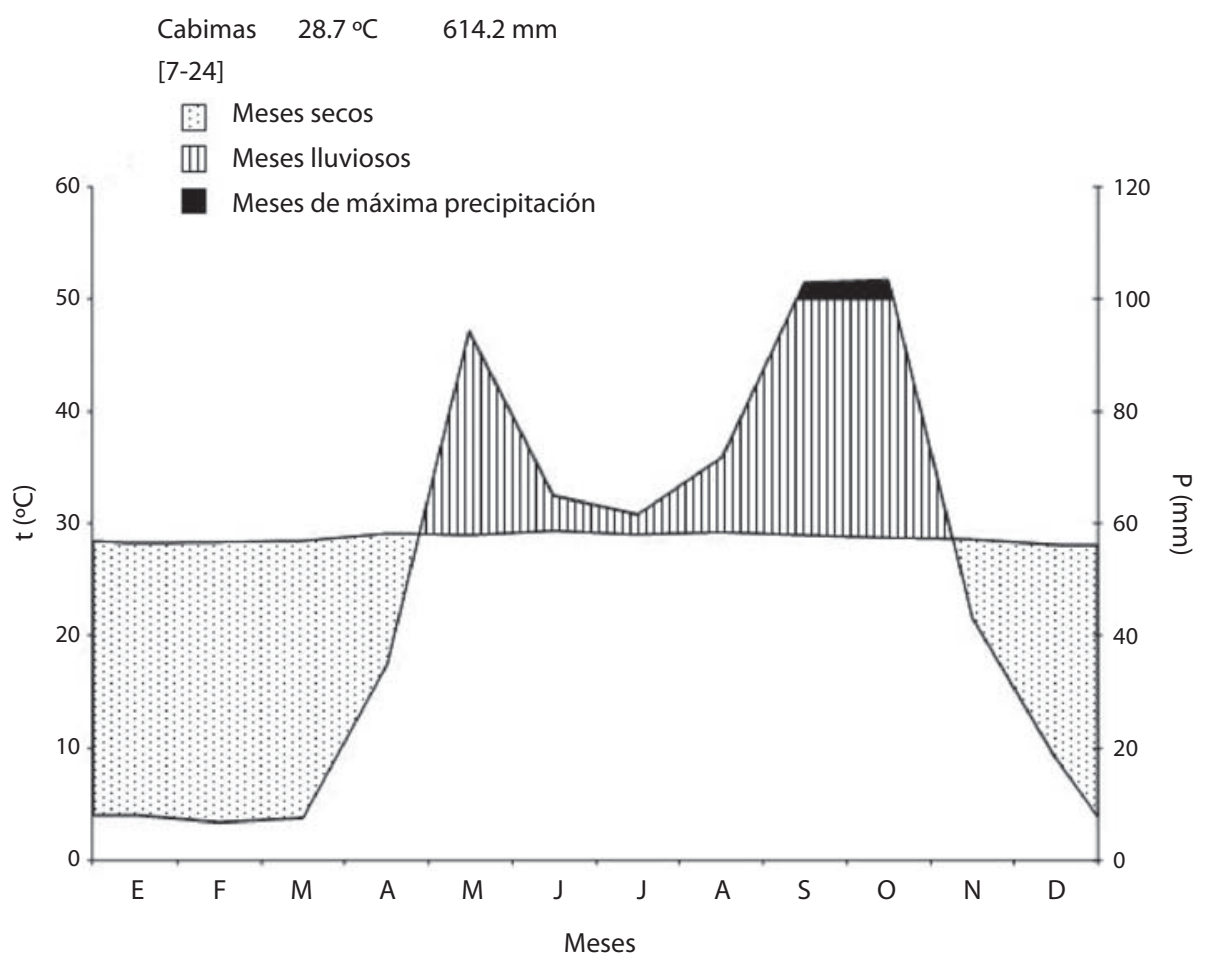

Fig. 2. Climograma del área de estudio.

Fig. 2. Climogram of study area. 
esta última unidad de muestreo se identificó y cuantificó el componente herbáceo, obteniendo 12 subparcelas en total. Esta metodología se aplicó debido a que la comunidad vegetal se encuentra agrupada conformando manchones, los cuales en su conjunto comprenden un área total de $7.178 .65 \mathrm{~m}^{2}$. También se realizaron recorridos para el reconocimiento de otras especies de la zona y así complementar los muestreos.

Las especies se identificaron con las claves de Aristeguieta (1973) y Little (1986) y a través de las comparaciones con las colecciones del Herbario Nacional (VEN), del Herbario de la Universidad del Zulia (HERZU) y de los Herbarios de Referencia de las Facultades Experimental de Ciencias y de Humanidades y Educación de la Universidad del Zulia.

Las muestras botánicas recolectadas se depositaron en el Herbario de la Universidad del Zulia (HERZU).

Perfil de Vegetación. Se elaboró un perfil de vegetación en un manchón seleccionado al azar; para lo cual se trazó una transecta de 40 $\mathrm{m}$ de largo por $2 \mathrm{~m}$ de ancho dentro del mismo (Noguera y Ruiz Zapata 2004). En el perfil se caracterizaron los diferentes estratos verticales (arbóreo, arbustivo y herbáceo) de acuerdo a la altura de los individuos de las diferentes especies.

\section{RESULTADOS}

El matorral xerófilo intervenido de Punta de Piedras está integrado por plantas, en su mayoría espinosas de hojas compuestas micrófilas, y algunas cactáceas. Se identificaron 45 especies, agrupadas en 22 familias, Cuadro 1, entre las cuales destacaron: las Poaceae (6), Mimosaceae (4), Boraginaceae (3), Cactaceae (3) Caesalpinaceae (3), Euphorbiaceae (3) y Fabaceae (3). De acuerdo al tipo biológico, hábito de vida o forma de vida, las 45 especies señaladas se distribuyen de la siguiente manera: 7 árboles de porte bajo, 10 arbustos, 2 sufrútices, 21 hierbas, 2 hemiparásitas y 3 trepadoras herbáceas, Cuadro 1.

La formación graminiforme o pastizal, localizada entre los manchones, se caracterizó por presentar áreas cubiertas por una vegetación rala, abierta y de porte bajo, con una altura media de $70 \mathrm{~cm}$. Los muestreos en esta formación arrojaron 21 especies de hierbas, de las cuales Aristida venesuelae Henr. 1928, A pittieri Henr. 1927 y Portulaca pilosa L. 1753 presentaron los mayores valores de abundancia y frecuencia relativa cuya sumatoria corresponde a $49.38 \%$ y $46.86 \%$, respectivamente, Cuadro 2.

Por otra parte, en los manchones del matorral xerófilo espinoso se destacaron dos estratos de vegetación (Fig. 3). El estrato herbáceo

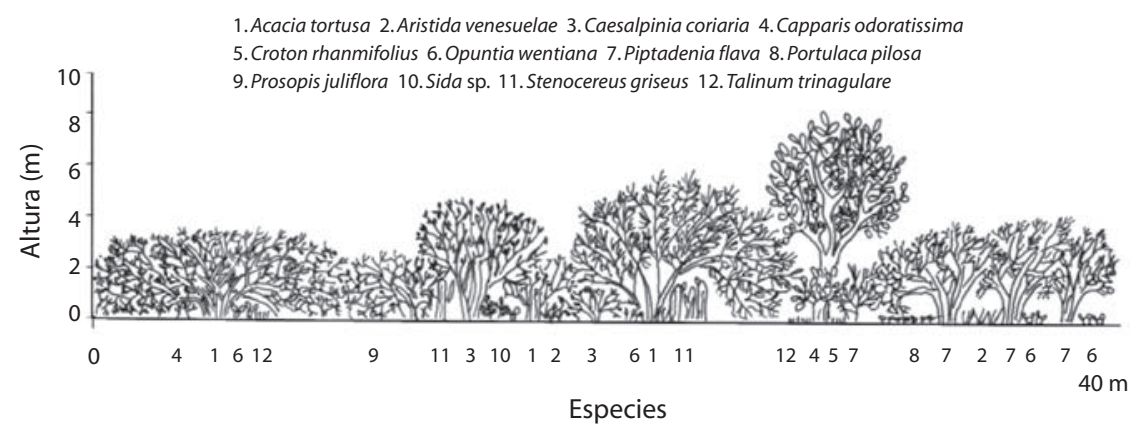

Fig. 3. Perfil de vegetación de un matorral xerófilo espinoso intervenido de Punta de Piedras, Municipio Miranda, Estado Zulia.

Fig. 3. Vegetation perfil of disturbed thorny xerophytic scrubland in Punta de Piedras, Miranda Municipality, Zulia State. 


\section{CUADRO 1}

Especies herbáceas, arbustivas y arbóreas de un matorral xerófilo espinoso intervenido de Punta de Piedras, Municipio Miranda, Estado Zulia

TABLE 1

Species herbaceous, shrub and tree of disturbed thorny xerophytic scrubland in Punta de Piedras, Miranda Municipality, Zulia State

\begin{tabular}{|c|c|c|}
\hline Familia & Especie & Hábito \\
\hline Amaranthaceae & Froelichia interrupta (L.) Moq. & Hierba \\
\hline \multirow[t]{3}{*}{ Boraginaceae } & Cordia alba (Jacq.) Roem. et Schult. & Árbol \\
\hline & Cordia curassavica (Jacq.) Roem. \& Schult. & Arbusto \\
\hline & Tournefortia angustifolia Ruiz F. Pavón & Hierba \\
\hline Bromeliaceae & Bromelia humilis Jacq. & Hierba \\
\hline \multirow[t]{3}{*}{ Cactaceae } & Opuntia wentiana Britton \& Rose & Arbusto \\
\hline & Rhodocactus guamacho (F. A. C. Weber) F. M. Knuth & Arbol \\
\hline & Stenocereus griseus (Haworth) F. Buxbaum. & Árbol \\
\hline \multirow[t]{3}{*}{ Caesalpiniaceae } & Caesalpinia coriaria (Jacq.) Willd & Árbol \\
\hline & Chamaecrista serpens (L.) Greene & Hierba \\
\hline & Senna atomaria (L.) Link & Árbol \\
\hline Capparaceae & Capparis odoratissima Jacq. & Árbol \\
\hline Convolvulaceae & Ipomoea $\mathrm{sp}$. & Hierba \\
\hline Cucurbitaceae & Momordica charantia $\mathrm{L}$. & Trepadora herbácea \\
\hline \multirow[t]{3}{*}{ Euphorbiaceae } & Croton rhamnifolius H.B.K. & Arbusto \\
\hline & Jatropha gossypifolia $\mathrm{L}$. & Sufrútice \\
\hline & Jatropha urens L. & Sufrútice \\
\hline \multirow[t]{3}{*}{ Fabaceae } & Stylosanthes sp. & Hierba \\
\hline & Zornia lasiocarpa A. R. Molina & Hierba \\
\hline & Zornia reticulata I. E. Smith & Hierba \\
\hline Flacourtiaceae & Casearia tremula (Griseb.) Wright & Arbusto \\
\hline \multirow[t]{2}{*}{ Malvaceae } & Herissantia crispa (L.) Brizicky & Hierba \\
\hline & Sida sp. & Hierba \\
\hline Martyniaceae & Craniolaria annua $\mathrm{L}$. & Hierba \\
\hline \multirow[t]{4}{*}{ Mimosaceae } & Acacia tortuosa (L.) Willd. & Arbusto \\
\hline & Mimosa sp. & Hierba \\
\hline & Piptadenia flava (Spreng.) Benth. & Arbusto \\
\hline & Prosopis juliflora (Sw.) DC. & Árbol \\
\hline Passifloraceae & Passiflora foetida $\mathrm{L}$. & Trepadora herbácea \\
\hline \multirow[t]{6}{*}{ Poaceae } & Anthephora hermaphrodita (L.) Kuntze & Hierba \\
\hline & Aristida pittieri Henr. & Hierba \\
\hline & Aristida venesuelae Henr. & Hierba \\
\hline & Cenchrus ciliaris L. & Hierba \\
\hline & Eragrostis ciliaris (L.) R. Br. & Hierba \\
\hline & Urochloa mollis (Sw.) Morrone et Zuloaga & Hierba \\
\hline \multirow[t]{2}{*}{ Polygonaceae } & Coccoloba sp. & Arbusto \\
\hline & Ruprechtia ramiflora (Jacq.) Meyer & Arbusto \\
\hline \multirow[t]{2}{*}{ Portulacaceae } & Portulaca pilosa $\mathrm{L}$. & Hierba \\
\hline & Talinum triangulare (Jacq.) Willd. & Hierba \\
\hline \multirow[t]{2}{*}{ Sterculiaceae } & Melochia tomentosa $\mathrm{L}$. & Arbusto \\
\hline & Ayenia magna $\mathrm{L}$. & Hierba \\
\hline Verbenaceae & Lantana cf. camara L. & Arbusto \\
\hline \multirow[t]{2}{*}{ Viscaceae } & Phoradendron mucronatum (DC.) Krug \& Urban & Hemiparásita \\
\hline & Phoradendron sp. & Hemiparásita \\
\hline Vitaceae & Cissus sicyoides $\mathrm{L}$. & Trepadora herbácea \\
\hline
\end{tabular}


CUADRO 2

Número de individuos, densidad, abundancia y frecuencia relativa de las especies herbáceas de la formación graminiforme del matorral xerófilo espinoso de Punta de Piedras, Municipio Miranda, Estado Zulia, Venezuela

TABLE 2

Number of specimens, density, abundance and relative frecuency of graminoid floristic components of disturbed thorny xerophitic scrubland in Punta de Piedras, Miranda Municipality, Zulia State, Venezuela

\begin{tabular}{|c|c|c|c|c|}
\hline Especie & $\mathrm{N}^{\mathrm{o}}$ de Individuos & D (ind/ha) & $\mathrm{AR}(\%)$ & FR $(\%)$ \\
\hline Aristida venesuelae & 16 & 13.333 & 19.75 & 19.16 \\
\hline Aristida pittieri & 14 & 11.667 & 17.28 & 15.62 \\
\hline Portulaca pilosa & 10 & 8.333 & 12.35 & 12.08 \\
\hline Froelichia interrupta & 7 & 5.833 & 8.64 & 6.88 \\
\hline Stylosanthes sp. & 4 & 3.333 & 4.94 & 6.88 \\
\hline Mimosa sp. & 4 & 3.333 & 4.94 & 5.21 \\
\hline Eragrostis ciliaris & 3 & 2.500 & 3.70 & 5.21 \\
\hline Sida sp. & 3 & 2.500 & 3.70 & 1.66 \\
\hline Cenchrus ciliaris & 2 & 1.667 & 2.47 & 3.54 \\
\hline Urochloa mollis & 2 & 1.667 & 2.47 & 3.54 \\
\hline Ayenia magna & 2 & 1.667 & 2.47 & 3.54 \\
\hline Chamaecrista serpens & 2 & 1.667 & 2.47 & 1.66 \\
\hline Anthephora hermaphrodita & 2 & 1.667 & 2.47 & 1.66 \\
\hline Craniolaria aпnиа & 2 & 1.667 & 2.47 & 1.66 \\
\hline Herissantia crispa & 2 & 1.667 & 2.47 & 1.66 \\
\hline Tournefortia angustifolia & 1 & 833 & 1.23 & 1.66 \\
\hline Zornia lasiocarpa & 1 & 833 & 1.23 & 1.66 \\
\hline Ipomoea sp. & 1 & 833 & 1.23 & 1.66 \\
\hline Momordica charantia & 1 & 833 & 1.23 & 1.66 \\
\hline Zornia reticulata & 1 & 833 & 1.23 & 1.66 \\
\hline Casearia tremula & 1 & 833 & 1.23 & 1.66 \\
\hline Total & 81 & 67.499 & 100.0 & 100.0 \\
\hline
\end{tabular}

(menor a $1 \mathrm{~m}$ de altura), localizado debajo de la copa del estrato arbustivo-arbóreo, está compuesto por, Sida sp. L. 1735, Talinum triangulare (Jacq.) Willd. 1799, Cenchrus ciliaris L.1771, A. venesuelae, A pittieri y Bromelia humilis Jacq. y plántulas de Capparis odoratissima Jacq. 1797, Opuntia wentiana Britton \& Rose 1919, Stenocereus griseus (Haworth) F. Buxbaum. 1961, Jatropha gossypifolia L. L. 1753, Cordia curassavica (Jacq.) Roem. \& Schult. 1819 y Croton rhamnifolius H.B.K. 1803 (Fig. 3). Los componentes del estrato herbáceo predominaban durante los meses de lluvia (Noviembre 2005 y Mayo 2006) (Fig. 2).

El estrato arbustivo-arbóreo, por otra parte, está constituido por sufrútices, arbustos y árboles bajos con una altura media de $2.63 \pm 1.37 \mathrm{~m}$. En éste se encontraron 18 especies, de las cuales los arbustos constituyen el grupo que presenta el mayor número (9); 7 corresponden a árboles de bajo porte (arbolitos) con troncos ramificados desde la base y 2 a sufrútices. Las especies de arbustos que obtuvieron la mayor abundancia relativa fueron $O$. wentiana, Piptadenia flava (Spreng.) Benth. 1875 y J. gossypifolia con 32.82, 15.60 y $15.14 \%$, respectivamente, cuya sumatoria representa un 63.56\%, Cuadro 3, (Fig. 3).

\section{DISCUSIÓN}

El matorral xerófilo espinoso de Punta de Piedras se caracteriza por presentar una 
CUADRO 3

Número de individuos, densidad, abundancia relativa y altura de las especies sufrútices, arbustivas y arbóreas de los manchones de vegetación del matorral xerófilo espinoso de Punta de Piedras, Municipio Miranda, Estado Zulia, Venezuela

TABLE 3

Number of specimens, density, relative abundante and height of species subshrub, shrub and tree of vegetation patches of disturbed thorny xerophitic scrubland in Punta de Piedras, Miranda Municipality, Zulia State, Venezuela

\begin{tabular}{lcccc}
\multicolumn{1}{c}{ Especie } & $\mathrm{N}^{\mathrm{o}}$ de Individuos & $\mathrm{D}(\mathrm{ind} / \mathrm{ha})$ & AR $(\%)$ & Altura $(\mathrm{m})$ \\
Opuntia wentiana & 516 & 719 & 32.82 & $0.86 \pm 0.23$ \\
Piptadenia flava & 245 & 341 & 15.60 & $3.02 \pm 1.91$ \\
Jatropha gossypifolia & 238 & 331 & 15.14 & $1.26 \pm 0.43$ \\
Capparis odoratissima & 184 & 256 & 11.70 & $3.49 \pm 1.69$ \\
Croton rhamnifolius & 95 & 132 & 6.04 & $1.45 \pm 0.79$ \\
Acacia tortuosa & 66 & 92 & 4.20 & $3.58 \pm 1.02$ \\
Prosopis juliflora & 51 & 71 & 3.24 & $4.22 \pm 1.42$ \\
Caesalpinia coriaria & 47 & 65 & 3.00 & $3.94 \pm 0.94$ \\
Stenocereus griseus & 40 & 56 & 2.54 & $1.72 \pm 1.51$ \\
Cordia curassavica & 39 & 54 & 2.48 & $1.48 \pm 0.44$ \\
Jatropha urens & 25 & 35 & 1.58 & $1.02 \pm 0.48$ \\
Melochia tomentosa & 7 & 10 & 0.45 & $1.37 \pm 0.41$ \\
Senna atomaria & 6 & 8 & 0.37 & $4.8 \pm 1.54$ \\
Lantana cf. camara & 5 & 7 & 0.32 & $1.03 \pm 0.23$ \\
Coccoloba sp. & 2 & 3 & 0.13 & $2.26 \pm 032$ \\
Cordia alba & 2 & 3 & 0.13 & $4.75 \pm 0.75$ \\
Rhodocactus guamacho & 2 & 3 & 0.13 & $4.4 \pm 0.1$ \\
Ruprechtia ramiflora & 2 & 2.189 & 100.0 & $2.72 \pm 0.35$ \\
Total & 1.572 & & & $2.63 \pm 1.37$
\end{tabular}

vegetación semiárida de espinar costero (Huber y Alarcón 1988), que se corresponde con el régimen de precipitación pluvial fuertemente estacional y de baja magnitud que predomina en la zona noroccidental de Venezuela (Fig. 2). Zambrano y Fuenmayor (1977) señalaron para el matorral xerófilo de Maracaibo, localizado en una zona semiárida del Occidente del Estado Zulia, especies con un marcado xerofitismo, predominio de plantas con espinas, hojas micrófilas y relativa abundancia de suculentas columnares. Las formas de vida de esta comunidad vegetal responden a los factores limitantes del ambiente como el déficit de humedad, las altas temperaturas y la pobreza nutricional del suelo (Zambrano y Fuenmayor 1977).

Sin embargo, la intervención antrópica, en el área de estudio, probablemete produjo la fragmentación de la comunidad en manchones de vegetación entre los cuales se extiende una formación graminiforme secundaria. Estos hallazgos se han encontrado en las zonas áridas de la Sierra Martín García, República Dominicana y en un remanente de bosque deciduo secundario del Valle de Caracas, Venezuela (López y Ramírez 2004, García et al. 2007). De igual forma, Noguera y Ruiz Zapata (2004) han informado la acción de los agentes antrópicos perturbadores (explotación forestal y actividades de pastoreo) sobre los bosques deciduos originales del Cerro La Gruta, próximo a la población de San Sebastián, estado Aragua (Fig. 4); éstos originaron la pérdida en la homogeneidad de dichas comunidades vegetales, y la aparición de herbazales hasta la conformación de un matorral.

En el estrato arbustivo-arbóreo de los manchones se destacó la presencia de las trepadoras 


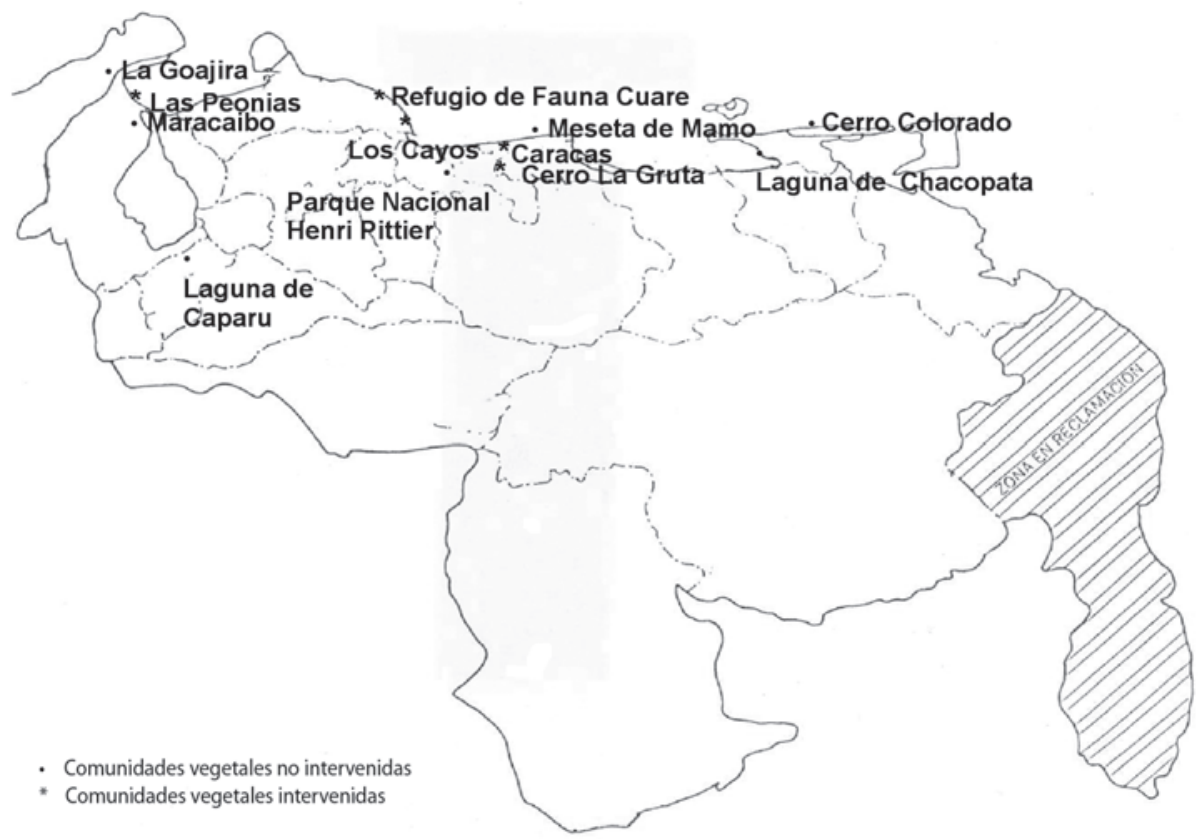

Fig. 4. Distribución de algunas comunidades xerófilas espinosas en Venezuela.

Passiflora foetida L. 1753, Momordica charantia L. 1753 y Cissus sicyoides L. 1759, como enredadera herbácea sobre arbustos espinosos, así como también de algunas hemiparásitas de la familia Viscaceae, Cuadro 1. Vera et al. (2005) han señalado para el matorral xerófilo espinoso de Punta de Piedras a Phoradendron sp. Nutt. 1847 y Phoradendron mucronatum (DC.) Krug \& Urban 1897, sobre las hospederas A. tortuosa y P.flava, con un promedio de 15 y 3 individuos de hemiparásitas/hospedera, respectivamente.

Las familias Poaceae, Mimosaceae, Boraginaceae, Cactaceae, Caesalpinaceae, Euphorbiaceae y Fabaceae constituyeron los taxones con mayor número de especies para el bosque xerófilo de la región de Las Peonías, estado Zulia, y en el ecosistema semiárido de la meseta de Mamo del estado Vargas, Venezuela (González 1980, Castillo et al. 1992), (Fig. 4.). En dichos trabajos, se mencionaron a Acacia tortuosa L. (Willd.) 1806, Aristida adscensionis L. 1753, A. venesuelae, Caesalpinia coriaria (Jacq.) Willd. 1799, Cenchrus brownii Roemer \& Schultes 1817, C. ciliaris, Cenchrus pilosus H. B. K. 1815, Mimosa arenosa (Willd.) Poir. 1810 y Prosopis julifora (Sw.) DC. 1825 (González 1980, Castillo et al. 1992). Todas estas familias mencionadas, que agrupan mayor cantidad de especies, para las comunidades vegetales de los estados Zulia y Vargas, así como también algunos géneros, se corresponden en gran parte con taxones señalados en esta investigación, Cuadro 1. Esto sugiere la correspondencia en la similitud de los componentes florísticos entre dichas formaciones vegetales.

Por otra parte, el matorral espinoso de Punta de Piedras mostró un bajo número de especies en comparación con las 133 informadas en el inventario del Monte Espinoso Tropical (comunidad vegetal no intervenida) del estado Vargas (Castillo et al. 1992), (Fig. 4). Este hecho se vinculó con el grado de intervención presente en el área de estudio, ya que se observó que esta comunidad vegetal está sometida a quemas periódicas, deposición de desechos sólidos, caminerías o senderos para el tránsito de las personas y asentamientos poblacionales en las zonas circunvecinas. 
Steyermark (1994) informó un total de 29 especies en el inventario florístico de la unidad de vegetación "Los Cayos", estado Falcón (Fig. 4), valor que se considera bajo. Asimismo, Silva y Espinoza (1995) describieron, para el Refugio de Fauna Silvestre "Cuare" del estado Falcón, un matorral xerófilo de poca altura, siempreverde, con una escasa cantidad de especies lo cual se atribuyó al establecimiento de complejos vacacionales, urbanizaciones y otras actividades vinculadas con la industria turística, lo cual altera y destruye el hábitat de las especies (Silva y Espinoza 1995). González 1980, por su parte, indicó 52 especies en la región de Las Peonías, estado Zulia, la cual ha sido sometida constantemente a la intervención del hombre a través de actividades como el sobrepastoreo de caprinos, la tala y quema indiscriminadas, la extracción de toneladas de tierra y el depósito de basura lo cual elimina extensas áreas de vegetación.

A pesar de que el matorral xerófilo espinoso de Punta de Piedras presenta intervención antrópica, los manchones mostraron rasgos fisonómicos (Fig. 3), comparables a los descritos para otras comunidades xerófilas espinosas, no intervenidas, de Venezuela (Fig. 4). La vegetación localizada en las cercanías de la Laguna de Chacopata, en la Península de Araya, estado Sucre constituye un arbustal xerófilo, que incluye un estrato herbáceo integrado por Opuntia caracasana Salm-Dyck 1850, J. gossypifolia, Cenchrus equinatus L. 1753 y Tribulus cistoides L. 1753 (Cumana et al. 2000). De igual forma, la localidad de la Laguna de Caparu, al sureste de San Juan de Lagunillas, estado Mérida presenta dos estratos: uno arbustivo, de $2 \mathrm{~m}$ de altura, formado principalmente por Cordia curassavica (Jacq.) Roem. \& Schult. 1819, C. rhamnifolius, Croton sp. L. 1754, J. gossypifolia, Opuntia spp. Mill. 1754; y otro herbáceo compuesto fundamentalmente por Lantana sp. L. 1753, Boerhavia erecta L. 1753, Sida sp., Evolvulus sericeus Sw. 1788, y Talinum sp. Adans. 1763 (Soriano et al. 1991). Igualmente, el Cardonal-Espinar del Parque Nacional Henri Pittier, estados Aragua y Carabobo, se caracteriza por poseer muy pocos elementos arbóreos y una evidente dominancia de arbustos espinosos (Fernández Badillo 1997).
Estos hallazgos sugieren que la fisonomía no se altera, en gran medida, por acción de los agentes antrópicos perturbadores.

En conclusión, el matorral xerófilo espinoso de Punta de Piedras presenta un bajo número de especies vegetales en comparación con otras comunidades xerófilas, no intervenidas, del país. Sin embargo, a pesar de la intervención antropogénica presente en esta comunidad, los manchones de vegetación mostraron rasgos fisonómicos análogos a los correspondientes a otras formaciones xerófilas espinosas no perturbadas en Venezuela.

\section{AGRADECIMIENTOS}

Al Centro de Investigaciones Biológicas de la Facultad de Humanidades y Educación de la Universidad del Zulia por ofrecer sus instalaciones y el apoyo logístico para el desarrollo de esta investigación. A Ángel Villarreal y a Darisol Pacheco por su valiosa colaboración en la identificación de algunas especies. A cuatro árbitros anónimos por sus comentarios y sugerencias que permitieron enriquecer y mejorar el manuscrito.

\section{RESUMEN}

Se determinaron los aspectos florísticos y fisonómicos de un matorral xerófilo espinoso intervenido de Punta de Piedras, municipio Miranda, estado Zulia, Venezuela. Se realizaron dieciséis muestreos quincenales, entre noviembre 2005 y junio 2006, para la recolecta, identificación y conteo de las especies tanto en el periodo lluvioso como en el seco. Los muestreos se llevaron a cabo en 10 manchones de vegetación con un área promedio de $718 \pm 706.24 \mathrm{~m}^{2}$, en una formación graminiforme en la que se emplearon tres parcelas de $20 \times 20\left(400 \mathrm{~m}^{2}\right)$, dividiéndose cada una de éstas en cuatro subparcelas de $20 \times 5\left(100 \mathrm{~m}^{2}\right)$ y a su vez, dentro de ellas, se delimitaron 12 subparcelas de $1 \times 1$. $\left(1 \mathrm{~m}^{2}\right)$, y también se realizaron recorridos para el reconocimiento de otras especies de la zona. El matorral xerófilo espinoso mostró evidencias de intervención antropogénica como quemas periódicas, deposición de desechos sólidos, caminerías para el tránsito de las personas y asentamientos poblacionales en las zonas circunvecinas; y presentó una vegetación semiárida de espinar costero característica y comparable a la señalada para otras regiones del país. Se identificaron 45 especies agrupadas en 22 familias, entre las cuales destacaron: las 
Poaceae (6), Mimosaceae (4), Boraginaceae (3), Cactaceae (3) Caesalpinaceae (3), Euphorbiaceae (3) y Fabaceae (3). La intervención antropogénica parece generar la aparición de manchones de vegetación arbustiva y arbórea y de una formación graminiforme o pastizal. En esta última, Aristida venesuelae, Aristida pittieri y Portulaca pilosa presentaron los mayores valores de abundancia y frecuencia relativa cuya sumatoria corresponde a $49.38 \%$ y $46.86 \%$, respectivamente; mientras que en los manchones se destacaron dos estratos: uno herbáceo menor a $1 \mathrm{~m}$, y otro estrato arbustivo-arbóreo de bajo porte $(2.63 \pm 1.37 \mathrm{~m}$ de altura media), conformado principalmente por Opuntia wentiana, Piptadenia flava y Jatropha gossypifolia, especies que presentaron las mayores abundancias relativas de 32.82, 15.60 y $15.14 \%$, respectivamente. El matorral xerófilo espinoso de Punta de Piedras presentó un bajo número de especies en comparación con otras comunidades xerófilas, no intervenidas, del país. Sin embargo, a pesar de la intervención antropogénica presente en esta comunidad, los manchones de vegetación mostraron rasgos fisonómicos análogos a los correspondientes a otras formaciones xerófilas espinosas no perturbadas de Venezuela.

Palabras clave: matorral xerófilo espinoso, bosque muy seco tropical, ecosistema perturbado, inventario florístico, fisonomía de vegetación, Venezuela, Zulia.

\section{REFERENCIAS}

Aristeguieta, L. 1973. Familias y géneros de árboles de Venezuela. Instituto Botánico, Dirección de Recursos Naturales Renovables, Ministerio de Agricultura y Cría. Edición Especial del Instituto Botánico. Gráficas Continente S.A. Caracas, Venezuela. 845 p.

Castillo, A., S. Gómez \& O. Moreno. 1992. Aspectos florísticos y fisionómicos de un ecosistema semiárido del Litoral Central, Municipio Vargas, Distrito Federal. Acta Biol. Venez. 13: 94-115.

Cumana, L., A. Prieto \& G. Ojeda. 2000. Flórula de la Laguna de Chacopata, Península de Araya, Estado Sucre, Venezuela. Saber 12: 25-33.

Ewel, J. \& A. Madriz. 1968. Zonas de Vida de Venezuela. Ministerio de Agricultura y Cría, Ediciones del Fondo Nacional de Investigaciones Agropecuarias. Editorial Sucre, Caracas, Venezuela 264 p.

Fernández Badillo, A. 1997. Zonas de Vida del Parque Nacional Henri Pittier, Venezuela. Rev. Fac. Agron. UCV (Maracay) 23: 249-270.

García, R., B. Peguero, T. Clase, A. Veloz, F. Jiménez \& M. Mejías. 2007. Flora y vegetación de las zonas áridas de la Sierra Martín García, República Dominicana. Moscosoa 15: 5-60.
González, E. 1980. Estudio preliminar de la vegetación del bosque xerófilo de la región de Las Peonías (Estado Zulia, Venezuela). Bol. Centro Invest. Biol. 14: 83-99.

Huber, O. \& C. Alarcón. 1988. Mapa de Vegetación de Venezuela. Ministerio del Ambiente y de los Resursos Naturales Renovables, Dirección General de Información e Investigación del Ambiente. Dirección de Suelos, Vegetación y Fauna. División de Vegetación, Escala 1:2.000.000 Base Cartográfica MOP. Oscar Todtmann Editores, Editorial Arte S. A., Caracas, Venezuela.

Little, E. L. 1986. Árboles comunes de Venezuela. Introducción general a la obra. Monografías 7.01 Serie Agro-Forestal. Consejo de Desarrollo Científico, Humanístico y Tecnológico, Consejo de Publicaciones, Universidad de los Andes, Mérida, Venezuela. 59 p.

López, M. J. \& N. Ramírez. 2004. Composición florística y abundancia de las especies en un remanente de bosque deciduo secundario. Acta Biol. Venez. 24: 29-71.

Ministerio del Ambiente y de los Recursos Naturales Renovables. 1980. Atlas de Venezuela. Dirección General de Investigación del Ambiente. Dirección de Cartografía Nacional. Caracas, Venezuela 329 p.

Noguera, E. \& T. Ruiz Zapata. 2004. Caracterización florística y fisonómica de tres parcelas ubicadas en el cerro "La Gruta", San Sebastián, Estado Aragua, Venezuela. Ernstia 14: 83-108.

Prieto, A., L. González \& P. Cornejo. 2001. Aspectos ecológicos de una población del cactus columnar Stenocereus griseus (Haw) F. Buxb en áreas xerofíticas del Cerro Colorado, Estado Sucre, Venezuela. Bol. Centro Invest. Biol. 35: 108-123.

Santana, R., F. Montagnini, B. Louman, R. Villalobos \& M. Gómez. 2002. Productos de bosques secundarios del Sur de Nicaragua con potencial para la elaboración de artesanías de Masaya. Revista Forestal Centroamericana $\mathrm{N}^{\circ} 38$ : 85-90.

Sarmiento, G. 1976. Evolution of arid vegetation in tropical America, p. 65-99. In D. W. Goodall (ed.) Evolution of desert biota. Univ. Texas, Londres.

Silva, A. \& F. Espinoza. 1995. Aspectos ecológicos del Cayo Noreste en el Refugio de Fauna Silvestre "Cuare", Estado Falcón, Venezuela. Acta Bot. Venez. 18: 21-52. 
Soriano, P., M. Sosa \& O. Rossell. 1991. Hábitos alimentarios de Glossophaga longirostris Millar (Chiroptera: Phyllostomidae) en una zona árida de los Andes venezolanos. Rev. Biol. Trop. 39: 263-268.

Steyermark, J. 1994. Flora del Parque Nacional Morrocoy. Editado por: Bruno Manara. Fundación Instituto Botánico de Venezuela y Agencia Española de Cooperación Internacional, Retolit C.A., Caracas, Venezuela. $415 \mathrm{p}$.

Tamayo, F. 1967. El espinar costanero. Bol. Soc. Ven. Cien. Nat. 27: 163-168.
Vera, A., O. López, S. Montes, K. Morillo \& Y. Ayala. 2005. Presencia de plantas hemiparásitas y hospederas en dos ecosistemas intervenidos del Municipio Miranda, Estado Zulia, Venezuela. Bol. Centro Invest. Biol. 39: $249-261$.

Zambrano, J. O. 1994. La sabana xerofítica: nueva denominación como tipo de vegetación en la Goajira venezolana. Rev. Fac. Agron. (LUZ) 11: 337-446.

Zambrano, J. O. \& E. Fuenmayor. 1977. El bosque muy seco tropical del Jardín Botánico de Maracaibo. Rev. Fac. Agron. (LUZ) 3: 79-87. 
